\title{
Treating Non-Hodgkin Lymphomas in Resource Limited Setting: 10 years of experience at the
}

university hospital

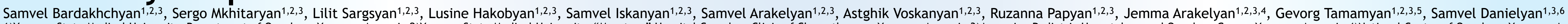

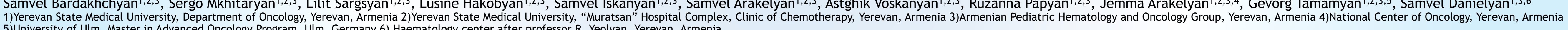

\section{Abstract}

Background: Non-Hodgkin lymphomas (NHL) are a heterogeneous group of lymphoproliferative malignancies responses to treatmem each other by behavior and various progress in the diagnosis and treatment of $\mathrm{NHL}$ in developed world, but the situation is different for the low and middleincome countries, and the data on this regard is limited.

Objective: The aim of the study is to describe distribution and treatment of various types of NHL in resource limited area and to estimate treatment outcomes

Patients and methods: For this retrospective, hospital-based study we identified patients from our patients' database with different types of primary diagnosed NHL who admitted and received treatment at the Clinic of Chemotherapy of "Muratsan" Hospital Complex of Yerevan State Medical University from 2008 to 2018. Overall 102 patients were identified and included in the study. $58(57 \%)$ were males. In the entire group $26(25.5 \%)$ were children ( $<18$ years old) and 76 adults

Results: In our cohort of patients' majority had diffuse large B-cell lymphoma - $46(45 \%)$. The main treatment approach used was chemotherapy \pm radiotherapy. 72 (70.5\%) patients completed planned chemotherapy cycles, 3 didn't receive any treatment, and the rest $27(26.5 \%)$ for different reasons dian't complete the whole course of chemotherapy. Mean number of chemotherapy cycles was 5 . The most often used chemotherapy regimen was CHOP/R-CHOP regimen among adults and NHL-BFM among children. From these 102 patients we have follow up information for 86 (84\%). Median follow up time was 3 years (range 0.1 to 9.4). At the time of follow-up 23 patients are not alive ( 21 due to cancer progression and 2 for causes other then cancer), 63 are alive (54 with no had disease recurrence). Conclusion: To the best of our knowledge this is the first descriptive study on NHL conducted in Armenia. Although the study has limitations (small and heterogeneous group), it shows that the treamer ou her of Further and larger studies are needed.

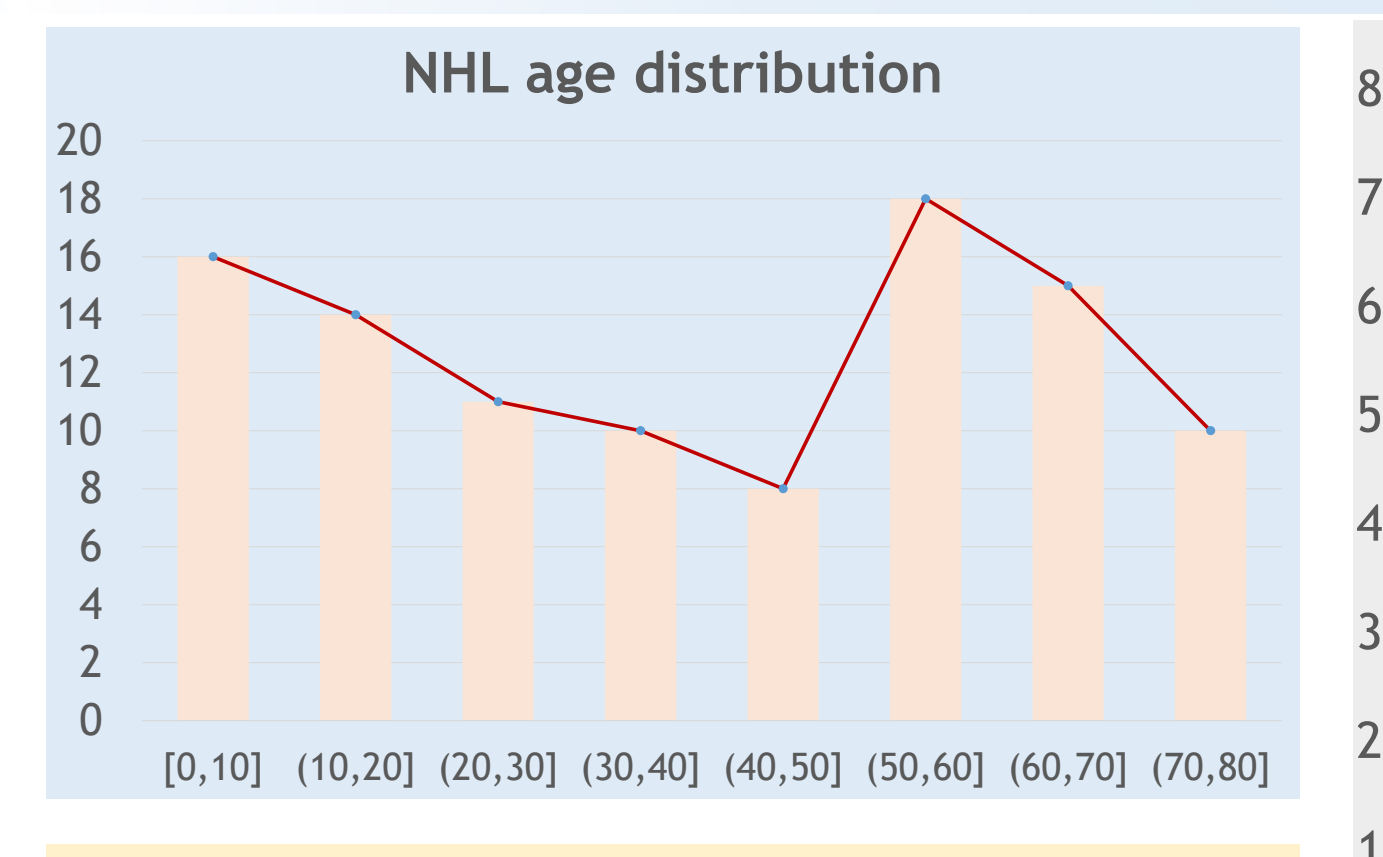

NHL distribution by type

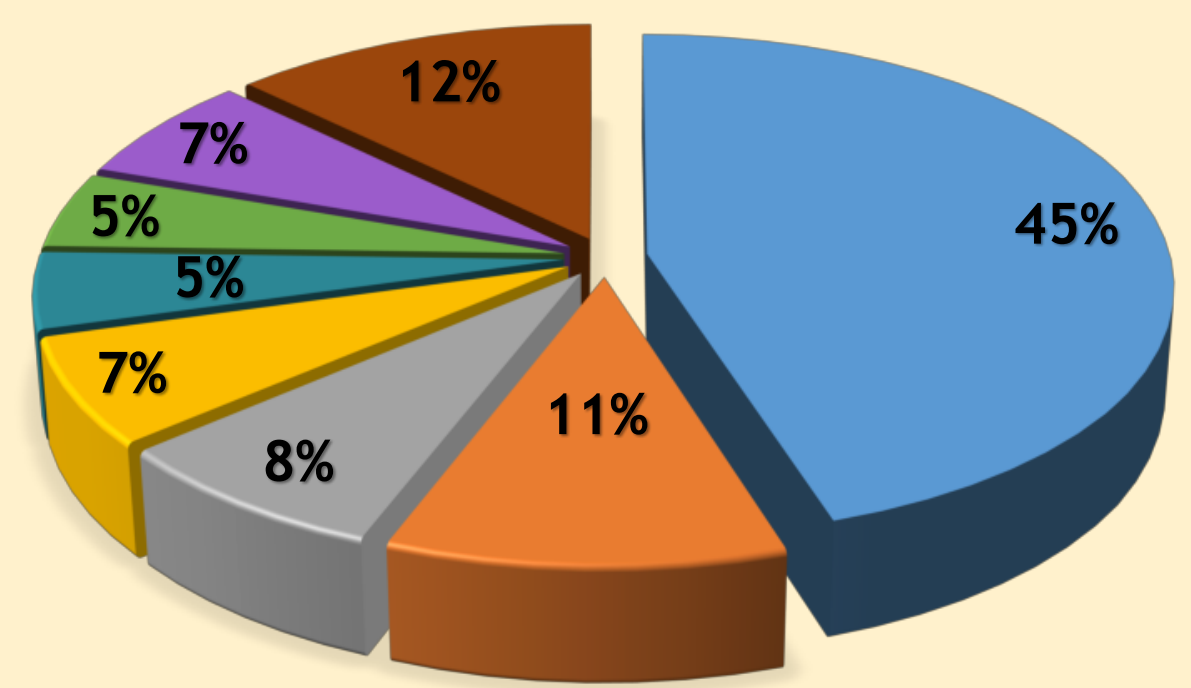

DLBCL T Iymphoblastic lymphomas

Marginal zone B lymphomas $\triangle \mathrm{ALCL}$

B I lymphoblastic lymphomas $\mathbf{Q}$ Burkitt's lymphomas

anot_Identified $\quad$ OOther

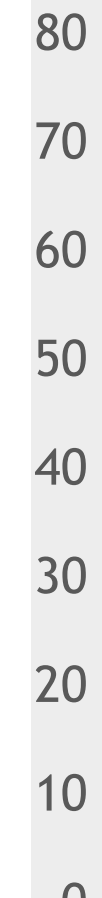

0

60
0
0
30
0
0
0

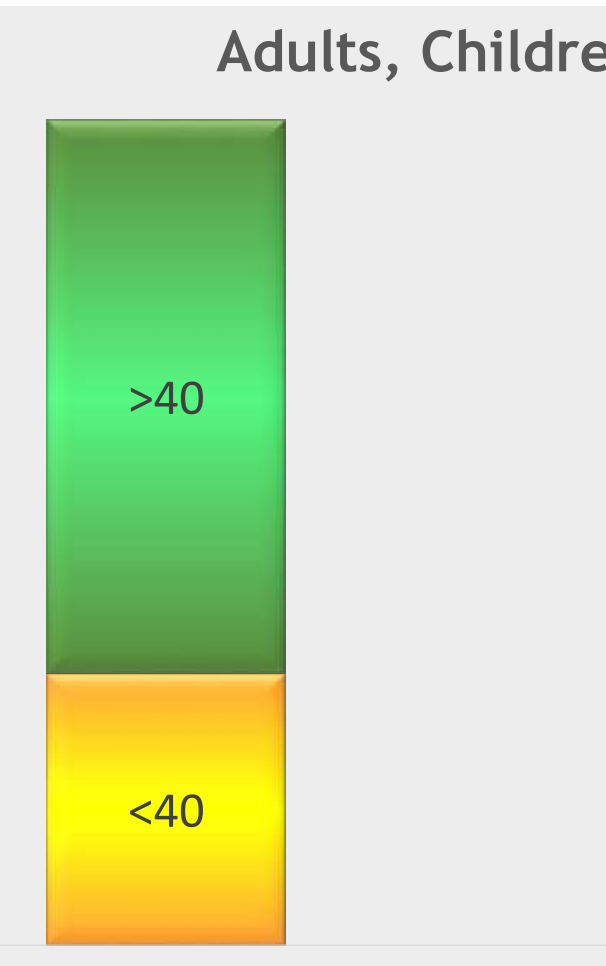

Adult

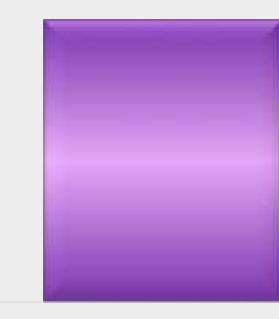

Child

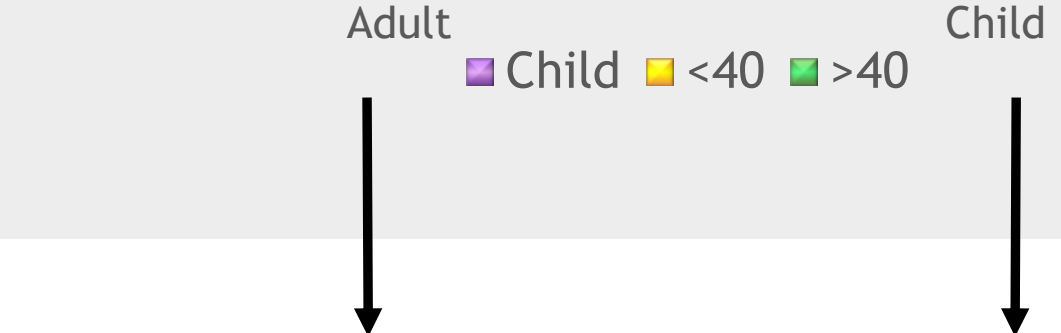

NHL among adults

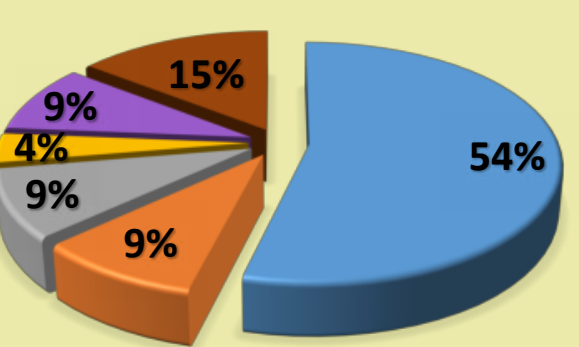

- T T lymphoblastic lymphomas

M Marginal zone B lymphomas

$\square \mathrm{ALCL}$

प Not identified

other
NHL among children

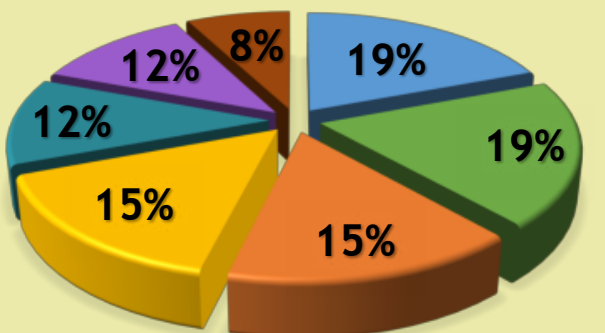

口DLBCL

G Burkitt's lymphomas

T T lymphoblastic lymphomas

$\triangle A L C L$

B B lymphoblastic lymphomas

Q Not identified

ather
Treatment results

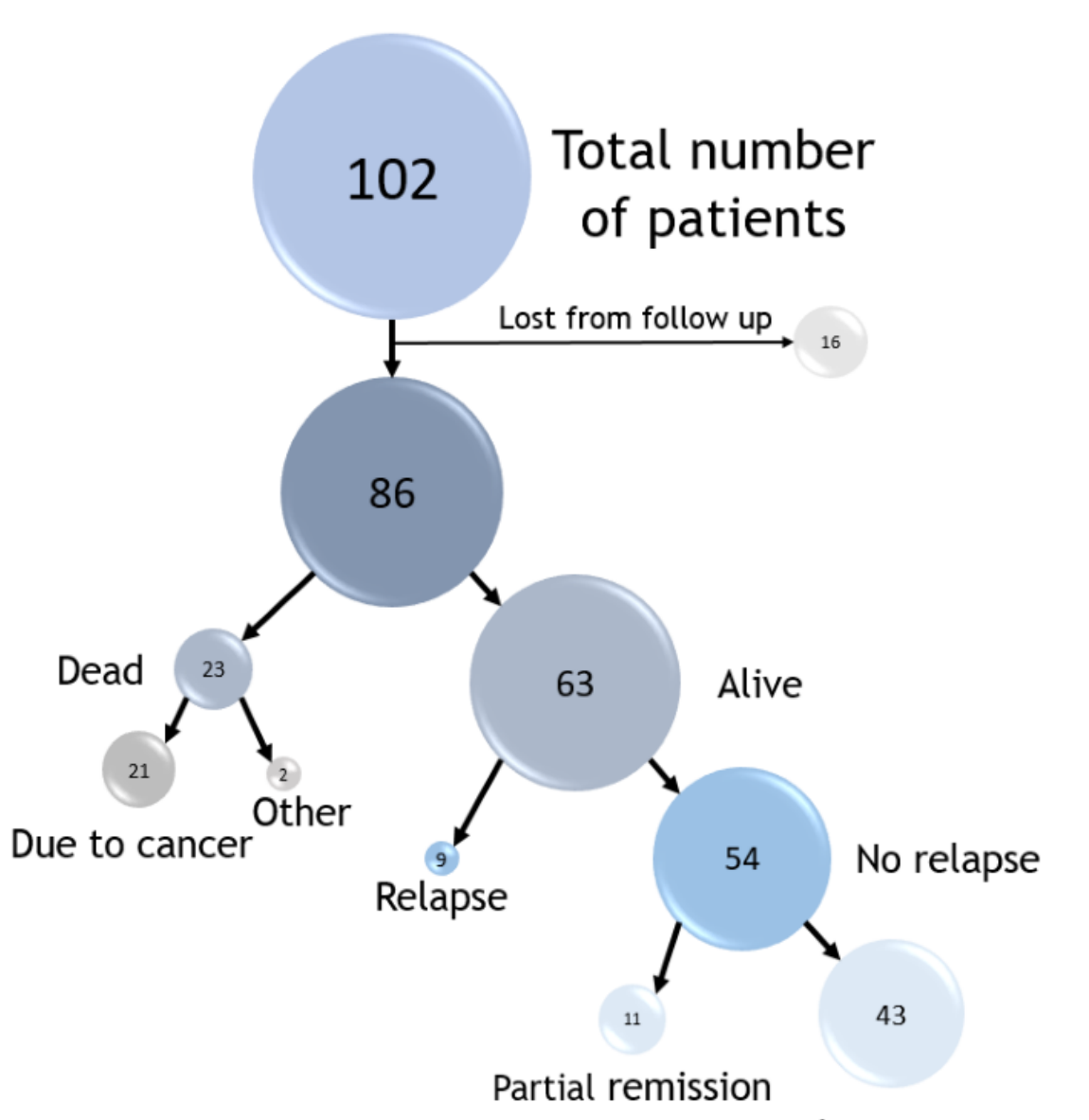

Complete remission

Results: From 102 patients we have follow up information for 86 (84\%). Median follow up time was 3 years (range 0.1 to 9.4). Only 72 completed planned chemotherapy cycles.

At the time of follow-up 23 patients are not alive (21 due to cancer progression and 2 for causes other then cancer), 63 are alive (54 with no relapse - from which 43 are in complete remission, 9 patients had disease recurrence).

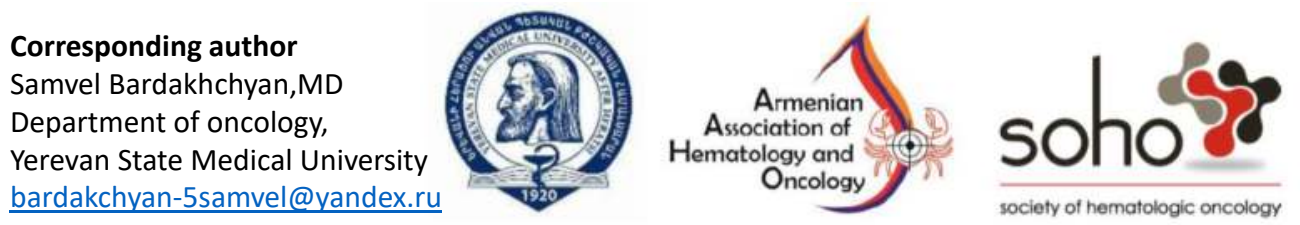

